\title{
Complutum
}

ISSN: 1131-6993

\section{Casas menstruales y liminalidad en sociedades orales: el bashali de la cultura kalasha (Pakistán)}

\author{
Matilde Carbajo $^{1}$
}

Recibido: 24/03/2021 / Aceptado: 13/08/2021

Resumen. El sesgo androcéntrico, que persiste en la investigación arqueológica y los discursos históricos sociales transmitidos por las instituciones encargadas del patrimonio cultural (principalmente museos), ha conllevado la fabricación y difusión de una imagen homogénea de las mujeres y sus instituciones. En consecuencia, a pesar de existir las casas menstruales en ambientes culturales radicalmente diferentes, se les ha dado a todas una misma razón de ser relacionada con la importancia simbólica de la sangre menstrual o/y su impureza. Recalcando la necesidad de profundizar en el conocimiento de esta institución desde la arqueología, en este artículo se analiza el caso de la casa menstrual kalasha (Pakistán) para concluir que, por su naturaleza liminal, funciona como motor de la dinámica social. También se contrasta la casa menstrual kalasha con otras provenientes de otros contextos geográficos con vistas a subrayar la diversidad y complejidad del fenómeno del aislamiento menstrual. Este trabajo no sólo contribuye a la recuperación de figuras y espacios invisibilizados, sino que permite cuestionar presupuestos en torno a la menstruación. Además, se recalca la necesidad del reconocimiento y la conservación de un patrimonio kalasha en femenino, ahora que dicha sociedad lucha por la supervivencia de su tradición cultural oral.

Palabras Clave: kalasha, casa menstrual, liminalidad, arqueología feminista.

\section{[en] Menstrual huts and liminality in oral societies: the Bashali of Kalasha Culture (Pakistan)}

Abstract. There is a pervasive male-centered bias in archaeological research and social discourses about history, which have been passed on through the institutions responsible for cultural heritage (mainly museums). It has often led to the construction and dissemination of a homogeneous representation of women and their institutions. Consequently, menstrual huts have been treated uniformly, despite existing in dramatically different cultural contexts. Previously, research has focused exclusively on the symbolic relevance of menstrual blood and/or its impurity, rather than on the women that inhabit these spaces. Aiming to deepen our knowledge of such institutions, I analyze the kalasha menstrual houses (Pakistan) and conclude that they are liminal spaces. They exist to bridge the divide between the known and the unknown and to set the social dynamic in motion. I do so from a cross-cultural perspective, illustrating the complexity of menstrual isolation. This work emphasizes the often overlooked cultural contribution of hidden figures and places, and is also relevant to Western menstrual assumptions. Additionally, I highlight the importance of recognizing kalasha feminine heritage, particularly in the context of their ongoing fight for preserving kalasha oral traditions and culture.

Key Words: kalasha, menstrual hut, liminality, feminist archaeology.

Sumario. 1. Introducción. 2. La cultura kalasha. 3. La casa menstrual kalasha. 3.1. Metodología. 3.2. Análisis macro. 3.3. Análisis micro. 3.3.1. El espacio habitacional. 3.3.2. El bashali. 3.3.3. La casa menstrual y el espacio habitacional. 4. La casa menstrual como espacio liminal. 5. Conclusión. Bibliografía.

Como citar: Carbajo, M. (2021): Casas menstruales y liminalidad en sociedades orales: el bashali de la cultura kalasha (Pakistán). Complutum, 32(2): 623-640.

\footnotetext{
$1 \quad$ Universidad Complutense de Madrid. Email: matica01@ucm.es
} 


\section{Introducción}

El patrimonio cultural -a imagen de la investigación histórica- se ha construido tradicionalmente desde una visión euro y androcéntrica (Smith 2008) legitimadora del patriarcado (Jiménez-Esquinas 2017). Esto se ha hecho a través de, entre otras cosas, la invisibilización de las mujeres, sus trabajos y acciones, su cultura material, tecnologías y espacios y, en definitiva, su contribución a la sociedad (Gonzalez Marcén et al. 2012). Así las mujeres han sido pasadas por alto no solo en los discursos, sino también en las reconstrucciones históricas de materiales divulgativos y educativos (Fernández Valencia 2005; García Luque 2015; 2016) y también en museos (Querol 2014; Navarro Rodríguez 2018; Jiménez-Esquinas 2021).

Estas reconstrucciones se han hecho siguiendo convenciones que recrean una serie de relaciones desiguales y privilegios (GiffordGonzalez 1993) tan normalizados que son solo evidentes cuando el género se incorpora como categoría de análisis (Smith 2008). Reflexiones y debates internacionales desde la arqueología feminista han llamado la atención sobre esto (Fries et al. 2017) y han avalado la producción de discursos "que asumiesen una responsabilidad social mucho mayor que la que puede suponer, por ejemplo, acertar en la fecha concreta" (Querol 2007: 211). Se han generado también imágenes alternativas y menos sesgadas sobre nuestro pasado, destacando en nuestro país el proyecto PastWomen. Por otro lado, desde el feminismo se ha dejado claro que, si bien la lucha por la representación es muy necesaria, no es suficiente y que "las exigencias de tipo cultural no pueden ir separadas de las exigencias socioeconómicas" sino que deben propiciar "la redistribución de los recursos y el acceso al poder político" (Jiménez-Esquínas 2017: 41).

La arqueología feminista, por tanto, va más allá de la mera búsqueda de mujeres en el pasado, tiene vocación transformadora e introduce el género como una herramienta crítica que permite cuestionar las relaciones de poder y dominación del sujeto histórico por excelencia (el hombre blanco) sobre las subalternidades, entre las que se encuentran las mujeres (Birriel Salcedo y Rísquez Cuenca 2016; Blake 2015; Méndez 2007). Una vez despatriarcalizado (Jiménez Esquinas 2016), el patrimonio se convierte en herramienta de transformación social (Arrieta Urtizberea 2017) y deja de estar al servicio del patriarcado (Jiménez-Esquinas 2017: 19).

Este trabajo busca inscribirse dentro de esta línea de acción. El estudio de la sociedad kalasha (Pakistán), con el foco en un espacio público (Rosaldo 1991) exclusivamente femenino como es la casa menstrual, pretende contribuir a la superación de la premisa de que las mujeres, en sociedades orales, quedan fuera de las instituciones responsables del desarrollo social. Además, permite combatir la idea de que las mujeres se han limitado a desempeñar funciones reproductivas o domésticas. Funciones que, por otro lado, no han sido consideradas dignas de ser incluidas en el análisis histórico-social. Por último, este trabajo permite contribuir a la ruptura de la universalización del sujeto mujer, que se desgaja de una homogeneización de lo femenino (Sánchez Romero 2019); un postulado que pervive en discursos científicos, divulgativos y en el imaginario popular incluso a pesar de la multiplicidad de experiencias, relacionadas con la pertenencia étnica, la clase social, la herencia histórica y cultural etc. (Engelstad 1991: 504). De forma análoga, a pesar de que la casa menstrual funcione como un dispositivo de control reproductivo (Méndez 2016) y la menstruación sirva para sustentar el sistema binario sexogénero (Guillo Arakistain 2013), la interpretación se ha limitado al "único" significado de la sangre menstrual: simbólicamente peligrosa (Buckley y Gottlieb 1988: 3-4)

En este sentido, la casa menstrual es uno de los espacios comunales al que menos tiempo le ha dedicado la investigación. Si bien es cierto que no constituye un fenómeno ubicuo, el aislamiento menstrual ocurre de forma casi universal (Frandsen 2007: 81), de hecho, la antropóloga Beverly Strassmann (1992: 90) afirma que "los tabúes menstruales están tan extendidos que es posible considerarlos universales culturales".

En multitud de ocasiones, dichos tabúes tienen una expresión material en forma de estructuras comunales especiales (las casas menstruales) que los hace susceptibles de ser estudiados desde nuestra disciplina. A pesar de esto, las casas menstruales están ausentes en la bibliografía arqueológica, con algunas excepciones en trabajos como los de Claassen (2011) o Faust y Katz (2017). Para Patricia Galloway (1998) y, más recientemente Anne Sherfield (2019), las razones de dicha ausencia radican en nuestro propio tabú sobre la menstruación: 
"así como las mujeres raramente menstrúan en la literatura occidental, la menstruación es generalmente ignorada cuando se trata de la reconstrucción arqueológica..." (Galloway 1998: 201).

El trabajo más exhaustivo del que podemos echar mano para entender cómo funciona una casa menstrual es, sin duda, el de Strassmann (Strassmann 1992; 1996; Strassmann et al. 2012). Sus escritos sobre las ponulu (cultura dogon (Mali)) dan cuenta de cómo funciona dicha institución en una sociedad regida por un sistema de pureza-impureza. En este contexto, el propósito de la casa menstrual es el de contener la impureza asociada al sangrado. Además, las ponulu sirven de dispositivo de control (Strassmann 1996); su cercanía a los espacios masculinos permite a los hombres estar al tanto de la vida reproductiva de las mujeres, "evitando gastar energía en vástagos con los que no están genéticamente relacionados, lo que es esencial en una cultura en la que el sistema de herencia es patrilineal" (ibid: 305 ).

Lo cierto es que la minuciosidad del análisis de Strassmann y lo abrumador de la relevancia de la ponulu para tener un conocimiento denso (sensu Geertz 1973) de la sociedad dogon, lejos de motivar el estudio de otros contextos, ha llevado a la extrapolación del funcionamiento de la ponulu (surgido en un ambiente cultural concreto) a otras realidades en las que existen casas menstruales, como, por ejemplo, la kalasha. En este sentido, merece la pena preguntarse ¿es homogéneo el fenómeno de las casas menstruales?, ¿funciona la casa menstrual de la cultura kalasha igual que la ponulu?

Para dar respuesta a la pregunta anterior, voy a aproximarme a la casa menstrual kalasha a través de una revisión bibliográfica y una reflexión, releyendo de forma crítica la literatura existente a la luz de esta nueva pregunta de investigación. Para ello, basaré mi análisis en los trabajos que diferentes antropólogos y antropólogas llevaron a cabo entre los sesenta y los noventa, con especial atención a las etnografías de Viviane Lièvre y Jean Yves Loude (1980; 1990; Loude y Lièvre 1984; Loude et al. 2018) y Wynne Maggi (2001).

\section{La cultura kalasha}

Los kalasha constituyen la última etnia no islámica de Pakistán. Son un pueblo ganadero que habita en poblados levantados a gran altura en tres escarpados valles del Hindukush (Himalaya pakistaní) llamados Birir, Rumbur y Bumburet. Kalashadesh (como se denomina al total del territorio kalasha) está situado en la región de Chritral, provincia de Khyber Pakhtunkhwa o KP (Pakistán) (figura 1). Su territorio limita con Afganistán al coincidir su límite más occidental con la frontera afgano-pakistaní que se identifica, grosso modo, con la línea Durand: el eje divisorio que fue establecido en 1893 por el Imperio Británico para definir el extremo más occidental de la -por entonces-India británica.

Esta línea consolidó la división de un área geográfica para la que recientemente se ha propuesto el nombre de Peristán (Cacopardo y Cacopardo 2001; Cacopardo 2016a). Este término hace referencia a la amplia región poblada por diferentes etnias preislámicas con cierta afinidad cultural (Jettmar 1961: 83). Previamente, el término utilizado era Kafiristán (tierra de los paganos), así aparece frecuentemente en la literatura. Este término (Kafiristán) es el nombre que los colonizadores islámicos dieron a la zona, al encontrarla poblada por gentes a las que denominaron "tribus kafires". Sin embargo, con la asimilación a la India Británica del territorio al este de la línea Durand (donde se

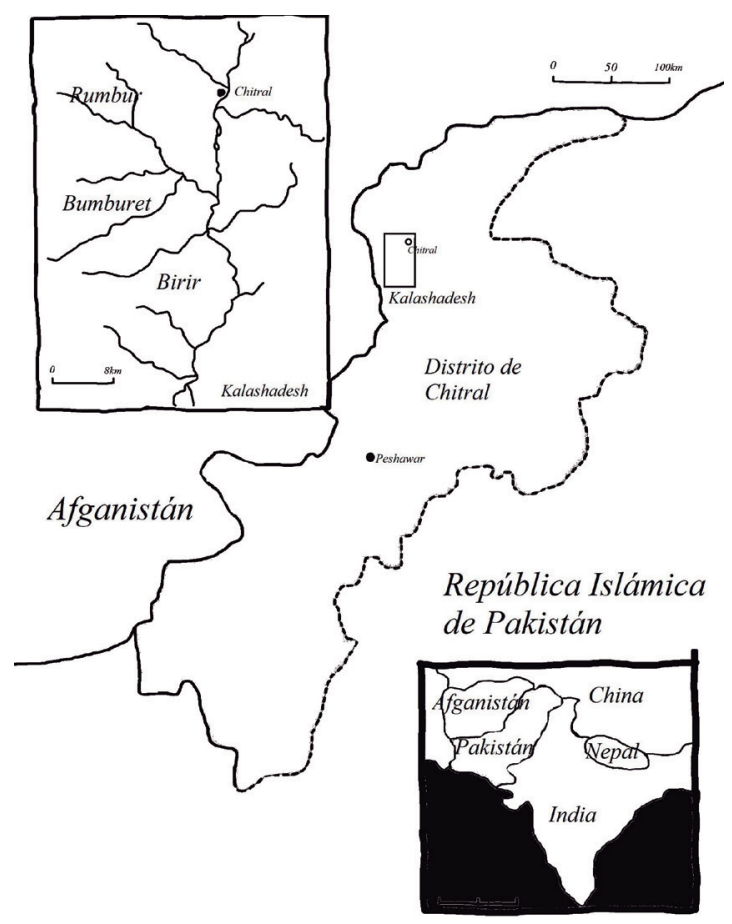

Figura 1. Mapa de Rumbur, Bumburet y Birir, los valles kalasha que conforman Kalashadesh, en su contexto geográfico general. Modificado de Loude et al. 2018. 
localiza, entre otras culturas, la kalasha), Kafiristán paso a hacer referencia exclusivamente al territorio correspondiente a la actual Nuristán (Afganistán) (Lièvre y Loude 1980; Loude y Lièvre 1984; Cacopardo y Cacopardo 2001; Cacopardo 2016a; 2016b).

La fecha para la llegada de los musulmanes a Peristán está en discusión; los autores han aportado cronologías que van desde el siglo VIII (Lièvre y Loude 1990) hasta siglo XVI (Cacopardo y Cacopardo 2001; Cacopardo 2016a). Lo que sí parece claro es que llegaron de forma progresiva, presionando a las llamadas tribus kafires que acabaron por convertirse al islam -a excepción de los kalasha- y ser absorbidas por las diferentes entidades políticas a lo largo del XVIII (Lièvre y Loude 1990; Cacopardo 2016a; Maggi 2001).

De la prehistoria de la región se sabe muy poco; se desconoce el origen de los pueblos preislámicos y las condiciones de su llegada a la zona y si existieron oleadas de colonización previas a la musulmana por parte de los estados budistas o el Imperio chino. Todo apunta, sin embargo, a que los pueblos del Hindu Kush se mantuvieron independientes política y religiosamente hasta la llegada de los musulmanes (Cacopardo y Cacopardo 2001: 28 - 30; Cacopardo 2016a: 72).

El pasado remoto de los kalasha es también incierto. Sin embargo, podemos afirmar con seguridad que están presentes en la zona desde la prehistoria y que su cultura ha ido pasando más o menos intacta de generación en generación a través de la transmisión oral del corpus dastur: el conjunto de reglas tradicionales kalasha (Maggi 2001: 45). Éstas fueron reveladas por los chamanes, figuras preeminentes de la comunidad. Entre ellas, está dictado el modo de vida: el pastoreo de la cabra de tipo transterminante complementado con una horticultura de cereal. Estas dos actividades rigen el calendario anual (Loude y Lièvre 1984; di Carlo 2007; Ali y Chawla 2019).

Es especialmente relevante la forma en la que la tradición kalasha ordenan el mundo: el territorio se clasifica según un complejo sistema de asociación de espacios, personas y actividades con los principios onjesta y pragata. Ambos dos están imbricados con el género: las mujeres, sus espacios y actividades son considerados pragata, mientras que la esfera masculina es onjesta. Así, por ejemplo, la ganadería de la cabra es tarea exclusiva de los hombres y considerada onjesta, mientras que el cultivo es una actividad pragata, responsabilidad de las mujeres. Esta división determina también el régimen de movilidad: mientras que los hombres gozan de mayor libertad de movimiento, asociada a la práctica de la trashumancia, las mujeres se desplazan en menor medida.

A pesar de la vigencia de onjesta y praga$t a$, la ordenación de la realidad no es binaria ni estática (Maggi 2001: 45). Si bien existen espacios absolutamente onjesta (los pastos de altura y altares de culto masculino) y espacios absolutamente pragata (la casa menstrual o bashali, que reúne a mujeres durante la menstruación y el parto), el resto de lugares queda entre medias. Así, por ejemplo, la zona de telares y el cementerio son considerados pragata aunque los hombres puedan acceder a ellos; y lo mismo ocurre con el área alrededor del fuego del hogar que, aunque se considera onjesta, puede ser ocupada por mujeres (Cacopardo y Cacopardo 2001: 69). De forma análoga, la mujer está en su estado más pragata justo después de dar a luz, sin embargo, durante el resto del tiempo será más o menos onjesta dependiendo de con quién se compare: una mujer que no tiene la menstruación será más onjesta que una mujer que está en el bashali por tenerla y ésta, a su vez, será más onjesta que una mujer que acaba de dar a luz y que, por tanto, también está en el bashali. Sin embargo, en condiciones normales, todas ellas serán más pragata que cualquier hombre (Maggi 2001: 44-72).

Si bien el funcionamiento de onjesta y pragata como principio ordenador está bien tratado a partir del trabajo de Maggi (2001), apenas existen datos sobre las instituciones pragata y, más concretamente, sobre el bashali. Esto es, en gran medida, debido a la traducción de onjesta y pragata como puro e impuro (sensu Douglas 1973), lo que ha llevado a mirar a través de los estándares de pureza e impureza. "Esto implica traslación de las connotaciones de "puro" e "impuro" a realidades que, quizás, no las compartan" (Maggi 2001: 47). Así, la asunción automática de que el ideal social se sitúa en lo puro, unido a la asociación de lo pragata con las mujeres, específicamente con la sangre menstrual y el parto, y a la existencia de la casa menstrual cuyos paralelos etnográficos más significativos (las casas menstruales dogon (Strassmann 1992; 1996; Strassmann et al. 2012)) están relacionados con la segregación de la impureza, llevaron a los pocos antropólogos que han trabajado sobre los kalasha y han tenido en cuenta la existencia del bas- 
hali (Robertson 1896; Schomberg 1938; Siiger 1956; Graziosi 1961; Palwal 1972 citado en Maggi 2001: 117) a interpretarlo como un contenedor de impureza asociado a lo femenino: "las mujeres eran consideradas impuras y, en tanto que portadoras de la impureza, debían estar en espacios impuros, especialmente durante la menstruación" (Magi 2001: 67). Sin embargo, la ordenación onjesta-pragata está lejos de funcionar según la lógica de lo puro y lo impuro, ya que la tradición kalasha busca el equilibrio entre los dos principios reguladores y la separación estricta de ambos (ibid.: 4649). En otras palabras, el ideal social kalasha no está en lo puro, sino en lo equilibrado.

Ahora bien, no debemos equiparar equilibrio con igualdad. Tal y como advierte Sherry Ortner (1974) para la lógica yin-yan del taoísmo chino, a pesar de que lo masculino y lo femenino sean igualmente importantes para garantizar la supervivencia grupal, el sistema puede seguir siendo desigual. Así, si miramos la estructura social kalasha, encontramos rasgos típicamente patriarcales: patrilinealidad, patrilocalidad, propiedad de la tierra en manos masculinas, importancia de los hijos varones $\mathrm{y}$, antiguamente, existencia de poligamia (Darling 1979; Maggi 2001). Además, mientras que la consideración "onjesta" a los varones no les supone ninguna restricción cotidiana, ser pragata es un enorme limitante de la movilidad, lo que constituye, por otro lado, una estrategia patriarcal histórica para impedir la individualización de las mujeres (Hernando 2002).

Así, al ser considerado el cuerpo de la mujer kalasha la materialización de lo pragata, ella puede alterar cualquier espacio con su mera presencia, rompiendo el equilibrio y trayendo terribles desastres: liberación de malos espíritus, deslizamientos de tierra, inundaciones, enfermedades, posesiones... (Maggi 2001: 47,50,63). En las mujeres recae, entonces, la garantía de la armonía en Kalashadesh. Sin embargo, al ser producto de una cultura oral, onjesta y pragata se identifican con lugares y momentos concretos cuya disposición es solamente cognoscible a través de la experiencia directa. Una mujer que salga de su contexto local puede causar por accidente enormes estragos.

\section{La casa menstrual kalasha}

Como ya hemos adelantado, el bashali es un edificio comunitario al que las mujeres kalasha acuden durante el periodo menstrual y al dar a luz. Es de naturaleza pragata, por eso, ningún hombre entra en él. Para abandonar el bashali, una vez acabado el periodo menstrual o tras el postparto, las mujeres deben lavarse con el agua del río que se encuentra en las inmediaciones. Esta regla se aplica no solo a las personas, sino también a la cultura material: cualquier cosa que entra en el bashali, debe ser lavada a conciencia antes de salir (Maggi 2001: 127).

Hasta donde sabemos, suele haber del orden de dos bashalis por valle, aunque algunos autores han sugerido que, en el pasado, el número pudo ser mayor, habiéndose reducido por causa de la presión colonial del islam (Graziosi 1961: 149). En cualquier caso y hasta la existencia de investigaciones arqueológicas en la zona, podemos tomar el valle de Rumbur como modelo general de disposición del asentamiento. En este valle, Nanga Dehar, el chamán más importante, estableció la primera comunidad en torno a los dos pilares de la cultura kalasha: el bashali (el lugar más pragata) y el altar de Sajigor (el lugar más onjesta) (Maggi 2001: 118). Este fue, por tanto, modelo y medida de los demás, orbitando en torno al bashali.

Para conocer el aspecto original de la casa menstrual, disponemos de un esquema de la planta del bashali de Bumburet (figura 2) dibujada por Paolo Graziosi (1961) quien, violando un tabú cultual, entró en el edificio durante su investigación en los años sesenta. De planta cuadrangular, el bashali sigue una tipología constructiva - a base de piedra y madera, con porche y hogar central - que no difiere mucho del resto de edificaciones kalasha. Es de suponer que, antes de la colonización europea, todas las casas menstruales compartían este mismo diseño arquitectónico, a pesar de que algunas de ellas no sean ya así (volveremos sobre esto en el siguiente punto). Sería interesante desarrollar investigaciones histórico-arqueológicas que permitiesen entender la forma en que la introducción de la cultura kalasha en el mundo globalizado ha modificado las casas menstruales de los diferentes valles.

En este sentido, las tensiones que conlleva el ser una minoría étnica dentro de la nación pakistaní, las disputas religiosas y los conflictos en torno al turismo, el desarrollismo económico y el patrimonio natural entre otros han causado enormes transformaciones en los últimos años, amenazando el estilo de vida tradicional kalasha (ver GHRD 2020. Para cono- 


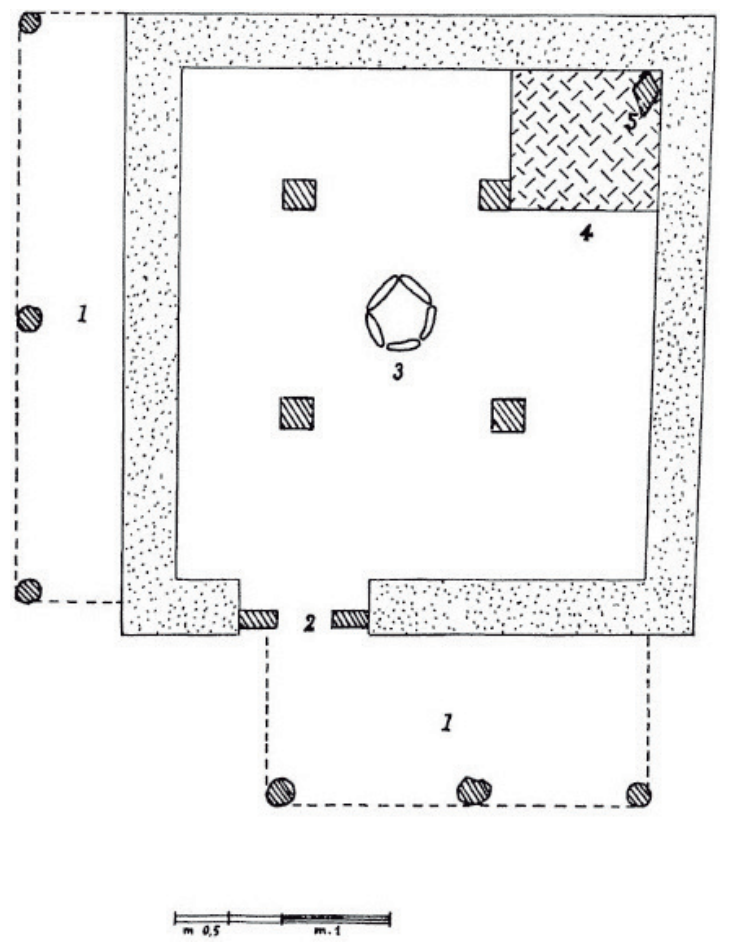

Figura 2. Planta del bashali del valle de Bumburet. 1-Porche exterior. 2-Entrada al interior del bashali. 3-Hogar central. 4-Altar a la diosa Dezalik. 5-Estatuilla de la diosa. Recuperado de Graziosi 1961.

cer la perspectiva indígena ver Parkes 1999; Malik y Waheed 2005; Junaidi 2017. Para un conocimiento histórico de la cuestión ver Naqvi 1996 y Rose 1992). A pesar de que analizar la posición que ocupa el bashali en medio de todas estas dinámicas (post)coloniales contemporáneas tiene gran interés, este trabajo se centra en el análisis de la casa menstrual según el corpus dastur que funciona de acuerdo a la lógica de la oralidad precolonial.

\subsection{Metodología}

Partiendo de la idea de que el análisis de la materialidad permite descubrir significados inconscientes, ocultos incluso para la gente que los ha producido, voy a analizar la espacialidad del bashali a escala macro y micro, sirviéndome para ello del esquema del valle de Rumbur (figura 3), dibujada por los antropólogos Jean-Yves Loude y Viviane Lièvre (1990); y la planta del bashali de Bumburet de los años sesenta (Figura 2).

Debemos tener en cuenta que un edificio singular -como es la casa menstrual-tiene ex- presiones espaciales y materiales cuyas características le hacen diferir de las estructuras domésticas (Ahlrichs et al. 2018). La contraposición de ambos contextos maximiza, por tanto, la capacidad de análisis. En consecuencia, se va a comparar la planta del bashali con la del espacio habitacional representado a través de un esquema de la casa kalasha recuperado de Lièvre y Loude (1984). El contraste entre ambos ayudará a perfilar la naturaleza del bashali.

A pesar de que la casa menstrual continúa en uso hoy en día, algunos bashalis no se corresponden ya con su arquitectura original. En el trabajo de Maggi (2001) se puede leer como en esa transición hacia el abandono de la oralidad y la basculación hacia el turismo como (principal) pilar económico, las costumbres en torno al bashali y la propia estructura física del edificio se han modificado, tanto por iniciativa europea (la segunda) como resultado directo de la agencia femenina kalasha (la primera). Así, en los últimos cincuenta años la sociedad kalasha ha iniciado un proceso de abandono de la oralidad que ha contribuido a la mayor individualización de algunas mujeres $\mathrm{y}$, por tanto, a cuestionar las restricciones impuestas por la tradición. En este mismo lapso de tiempo, han sido numerosas las remodelaciones de algunos bashalis hechas por orden de mujeres europeas y apoyo económico de ONGs y otras instituciones que buscaban adaptarlas a los estándares de comodidad occidentales.

Tomemos como ejemplo el bashali del valle del Rumbur. Antes de los años ochenta - en que se produjo una primera pequeña reforma habría tenido una planta similar a la de la casa menstrual de Bumburet (figura 2): una habitación cuadrada con hogar central. En el 94, se modificó de forma dramática cuando una adinerada mujer sueca encargó sustituir el hogar central por una chimenea, y añadir cocina $y$ baño, paredes de cemento, techo de metal y un muro perimetral, para proporcionar "privacidad" (Maggi 2001: 123). Hay que dejar claro, por tanto, que, a pesar de seguir en uso hoy en día, algunas de las casas menstruales de Kalashadesh no conservan ya su aspecto original.

\subsection{Análisis macro}

Rumbur, al igual que el resto de los valles kalasha, tiene una angosta forma en $\mathrm{V}$ en la que se acopla el tejido habitacional, compuesto por una serie de pueblos y diversas instituciones de uso común: el cementerio, los santuarios de 


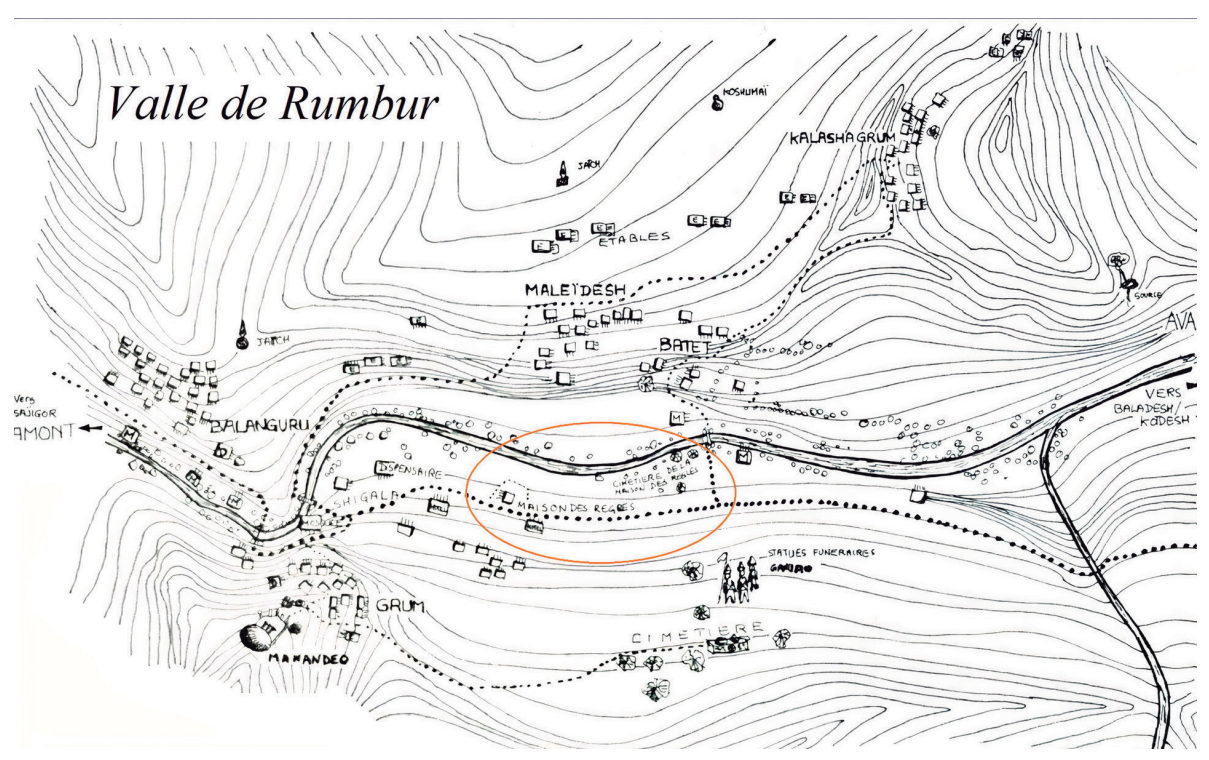

Figura 3. Valle de Rumbur. El área del bashali, incluyendo la casa menstrual y la necrópolis asociada, está señalada con un círculo. Adaptado de Loude y Lièvre 1990.

culto masculino y la casa menstrual. $\mathrm{Su}$ distribución es la que se recoge en la figura 3: en las altitudes medias se sitúan los poblados y el cementerio, en el fondo del valle se localizan el río y, paralelo a éste, el camino, única vía de comunicación. Asociado a los dos últimos se encuentra el bashali con su pequeña necrópolis. A una cota superior se localizan establos, pastos de día y altares de culto masculino; edificios que, por regla general, son de carácter onjesta (Parkes 1987).

La economía kalasha, como ya hemos apuntado, descansa sobre dos pilares principales: la horticultura de cereal (responsabilidad de las mujeres) y la ganadería caprina (responsabilidad de los hombres). Ambas implican una movilidad relativamente elevada un régimen habitacional estacional: la comunidad kalasha convivirá durante el invierno mientras que en las épocas más benignas los hombres irán a los pastos de altura; las mujeres, a residencias secundarias cerca de los campos de cultivo (Maggi 2001). Ahora bien, tanto en verano como en invierno, los periodos menstruales y el parto deben transcurrir en el bashali. Así, independientemente de la época del año, las mujeres deben desplazarse al fondo del valle una vez al mes dando lugar a una ocupación ininterrumpida de la casa menstrual cuyo emplazamiento - a una cota baja- permite el acceso durante la totalidad anual.

Este hecho contrasta enormemente con el dinamismo existente en el resto de ambien- tes: los santuarios y pastos de altura, que son inaccesibles en invierno; y el asentamiento principal y el secundario, que se combinan en una estrategia de poblamiento semianual. En este sentido, además de continuidad temporal, el bashali hace de centro aglutinante de la comunidad dispersa en época estival. La casa menstrual es, por tanto, un punto de anclaje, un espacio que proporciona estabilidad y continuidad a un paisaje femenino en continuo movimiento. Además, el bashali constituye un punto de encuentro, de intercambio de noticias y de relación para una comunidad de mujeres dispersa a lo largo del valle.

Además de continuidad espacio-temporal, el bashali es un referente identitario étnico, ya que es una institución que funciona a nivel de valle. Éste, ha sido tradicionalmente la máxima unidad geográfico-administrativa e identitaria. Así, aunque actualmente los kalasha se identifican como uno y tiendan a hablar con una sola voz, especialmente en acontecimientos nacionales e internacionales (ver Ali y Rehman 2001; Cacopardo y Cacopardo 2001; Parkes 2000), cada valle se consideraba independiente de los otros hasta momentos recientes. Evidencia de ello es que, en tiempos preislámicos, algunas etnias peristaníes se referían a los kalasha de una forma diferente, por ejemplo, llamaban "Kasua" a los habitantes de Bumburet y "Weru" a los de Birir (Cacopardo 2016a: 73). Según esto, se podría aventurar la existencia de una comunidad autoidentificada 
kalasha, con su etnónimo correspondiente, por cada bashali, sirviendo éste de punto de referencia étnico en tiempos precoloniales. Por supuesto, para afirmar esto con rotundidad, haría falta generar un corpus de evidencias que confirmara dicha hipótesis.

Como se ha señalado anteriormente, el ser extranjero en uno de los valles kalasha tiene enormes implicaciones para las mujeres. Maggi (2001: 44-71) documenta que aquella mujer que se casa fuera de su valle, o que va de visita, se siente temerosa e insegura: al no conocer la disposición de los espacios pragata y onjesta podría alterar el equilibrio accidentalmente. El bashali, sin embargo, ayudaría a aliviar esta angustia gracias a su naturaleza tipológica y su carácter inmutable: el bashali es universal dentro del mundo kalasha.

Además de ser un edificio estable que puede servir de ancla y brújula, la casa menstrual es un referente paisajístico: es el primer elemento visible en el acceso al valle y lo último que se divisa al salir. Es, de igual modo, inevitable - al menos visualmente - cuando se viaja de un pueblo a otro o si se acude al río. Se trata, en definitiva, de un lugar poco privado. Aquí radica la principal diferencia con las casas menstruales dogon. Lejos de estar aislado, semi-escondido o ser poco accesible, el bashali se encuentra en el centro de la actividad diaria. Tradicionalmente exento de barreras físicas y levantado en el terreno plano, la casa menstrual es completamente visible desde el camino y viceversa y permite la comunicación con cualquiera que pase por allí.

Además de con el camino principal, el bashali está asociado con el río y con la necrópolis secundaria. Estos dos elementos confieren a la casa menstrual un carácter muy especial. No es necesario extenderse en la importancia simbólica del agua; su naturaleza fluida y su capacidad de transformación y limpieza la convierten en un aliado ideal para lo ritual, lo espiritual y lo sacro (Oestigaard 2005; 2011). En el caso que nos ocupa, el agua permite la transición entre el bashali y la vida normal, actuando como elemento reintegrador. Sin embargo, la reintegración no siempre es posible. Si se produce la muerte de alguna mujer en el parto, o de ésta y/o el bebé durante el posparto, la norma dicta que se entierre a madre y/o criatura en un lugar especial, que no se frecuenta nunca y del que no se habla: la necrópolis reservada para los difuntos del bashali, situada a poca distancia de la casa menstrual. En estas circunstancias, las personas que se entierran (el rito de enteramiento habitual es la exposición del cadáver en sarcófagos de madera abiertos) lo hacen sin ceremonia alguna (Maggi 2001: 158).

Además de con la muerte, el bashali está asociado con la vida. Como ya se ha mencionado, tradicionalmente todas las mujeres deben dar a luz en la casa menstrual y, por consiguiente, todos los habitantes del valle nacen allí. Paolo Graziosi (1960), en su artículo The Wooden Statue of Dezalik, a Kalash Divinity, recoge la siguiente anécdota: cuando está realizando un censo en Bumburet para hacer un estudio demográfico, busca documentar la localidad natal de cada individuo. Sin embargo, no es capaz de registrar nacimiento alguno en los pueblos, ya que todos afirman haber venido al mundo en el bashali. Así, podemos decir que todo kalasha nace dos veces: una primera en la casa menstrual y una segunda cuando, tras haberse lavado, entra a formar parte de la sociedad kalasha. La asimilación del bebé no es (solamente) una forma simbólica de integración mediante el agua, sino que implica un cambio espacial literal.

\subsection{Análisis micro}

\subsubsection{El espacio habitacional}

Los espacios habitacionales, de forma cuadrangular, hogar en el centro y con una única abertura en el techo amén de la puerta (Loude et al. 2018) cuentan con un porche donde se desarrolla la vida diaria, sobre todo durante la época de buen tiempo (Magi 2001: 59). Para edificarlos, además de madera y piedra locales, los kalasha aprovechan la orografía levantando las casas unas sobre otras adosadas a las laderas de las montañas. Esto resulta en un paisaje habitacional escalonado donde el porche de algunas casas hace de techo de las que están por debajo (figura 4). Los accesos y desplazamientos domésticos principales se hacen, en consecuencia, en sentido vertical, mediante escaleras o aprovechando los salientes de las piedras y las vigas en los lienzos (Loude et al. 2018).

La vivienda está repartida en cuatro espacios tal y como se puede observar en la figura 5. El primero de ellos, propiamente habitacional, está dividido en dos estancias: una interior y un porche de madera que concentra la mayor parte de la actividad diurna. Éste está equipado con un hogar destinado al procesado de alimentos y un molino de mano. De su 


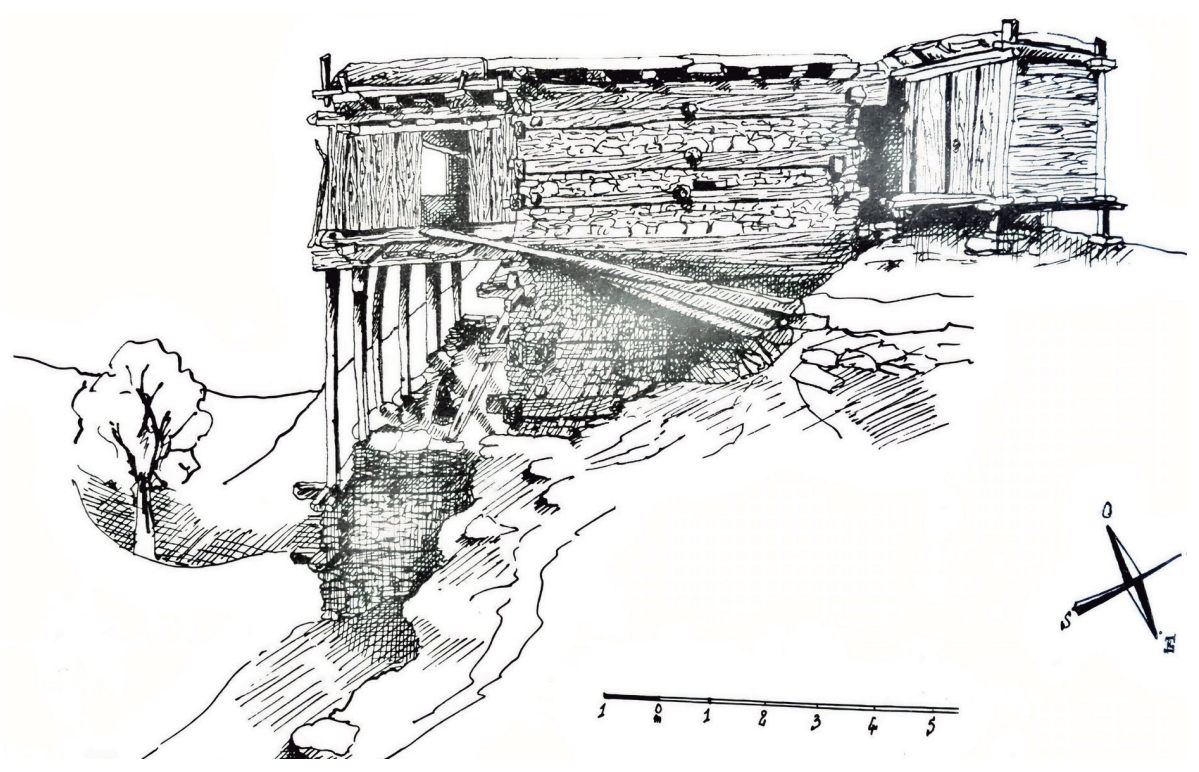

Figura 4. Dibujo del exterior de una casa kalasha construida en pendiente, adosada a la ladera de la montaña. La superposición de varias de ellas resulta en un pasaje aterrazado. Recuperado de Loude y Lièvre 1990.

espacio se dispone según la necesidad: secar la ropa o la comida, cocinar, reunirse o recibir visitas (Loude y Lièvre 1984: 75-83). Este espacio permite gran visibilidad del valle y a la vez aporta mucha privacidad debido a la construcción escalonada y lo empinado de la pendiente; dos elementos que le confieren cierto carácter de plataforma.

La estancia principal, en cambio, está rígidamente estructurada. Se organiza en torno a dos elementos: el hogar central y el altar doméstico, ambos alineados entre sí y con la puerta. El altar, colocado contra la pared del fondo, está dedicado a Jeshtak, diosa de la familia y el linaje. A la misma divinidad está consagrado un santuario de acceso general situado en medio del poblado, donde se celebran las principales fiestas, ritos y reuniones; este santuario no se clasifica como onjesta o pragata (Cacopardo y Cacopardo 2001: 71).

Sin embargo, entre el altar doméstico y el hogar central existe una línea que sí se considera onjesta: el onjesta-wao, que rompe con la disposición circular de la vivienda. Maggi (2001: 57-58) y Loude y Lièvre (1984: 66-68), dan cuenta de las enormes repercusiones que tiene dicha línea en el uso del espacio ya que, al ser onjesta, las mujeres no pueden cruzarla. Así, mientras que para los hombres la estancia es circular, para las mujeres tiene forma de "U" (Maggi 2001: 57-58). Por último, encontramos la zona de despensa. Además de en gra- neros exteriores (Loude y Lièvre 1984: 75-83), los kalasha almacenan dentro de la casa, bien en una habitación independiente, bien contra la pared del fondo de la principal, siempre respetando el onjesta-wao.

\subsubsection{El bashali}

A pesar de que la tipología tradicional del bashali registra marcadas diferencias con la del espacio habitacional, comparte también algunos rasgos: el diseño cuadrangular, el porche, el hogar central en la sala principal y una concepción espacial cuatripartita. En la entrada encontramos la primera desemejanza respecto a la casa, donde el acceso es muy dificultoso. En cambio, el bashali es fácilmente permeable debido a las condiciones de emplazamiento.

Por su parte, el porche, en ambos casos el ambiente cotidiano principal, registra grandes diferencias en cuanto a la visibilidad. En el bashali las mujeres están enormemente expuestas y a la vez, tienen gran visibilidad de lo que pasa fuera. De hecho, en el caso del bashali que Graziosi documentó, existe un segundo porche que aporta mayor superficie de uso y, a su vez, mayor visibilidad.

Por otro lado, el porche de la casa es una zona poco estructurada. Aunque existen en ella elementos que fuerzan la funcionalidad de ciertas áreas, suelen ser la actividad del momento la que dicta la disposición de objetos y perso- 


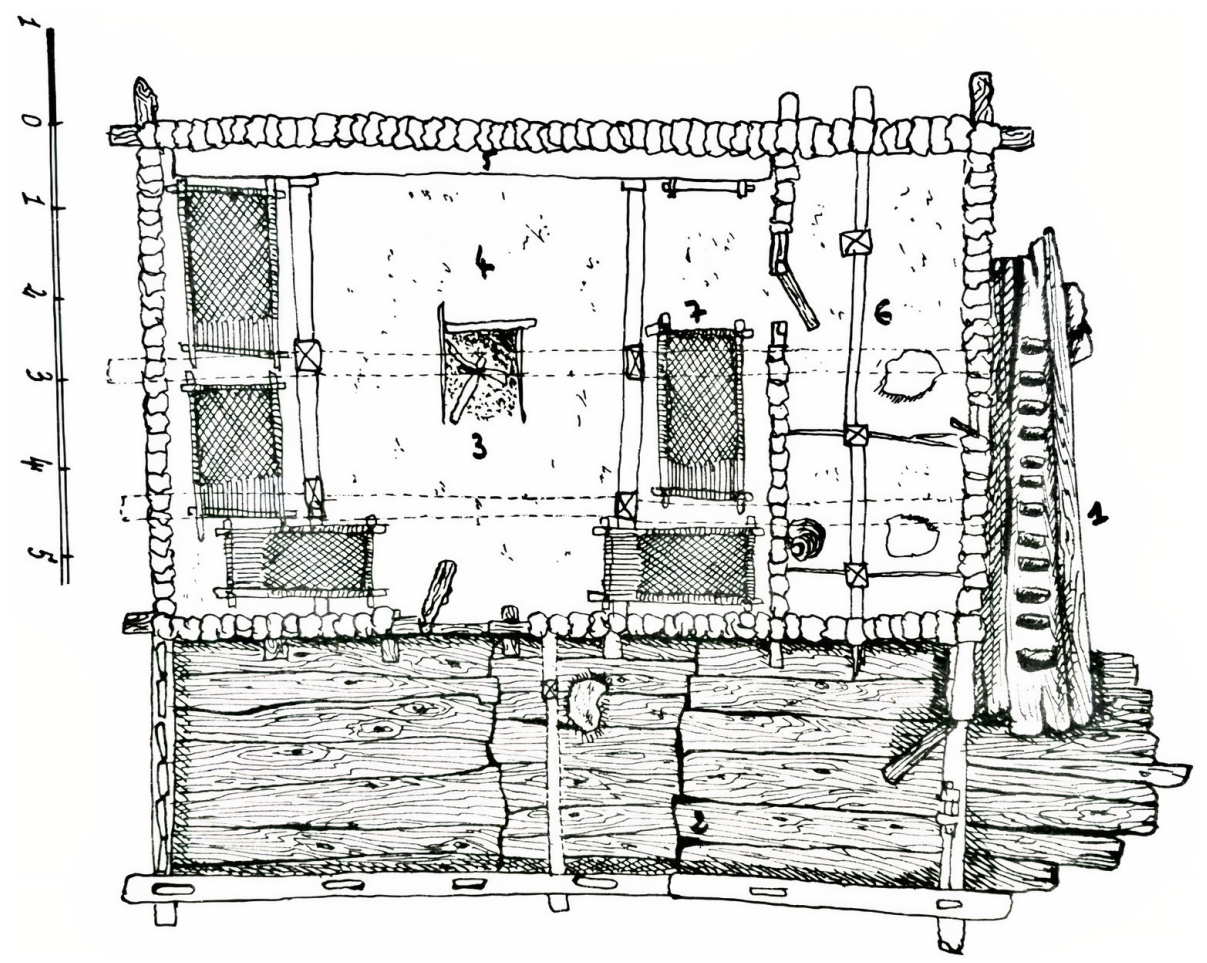

Figura 5. Planta del espacio habitacional kalasha, repartido en cuatro espacios: la escalera de acceso, el porche exterior, la habitación principal y la zona de almacenaje. 1-Escalera lateral.

2-Porche. 3-Hogar central. 4-Onjesta-wao. 5-Altar doméstico. 6-espacio de almacenaje.

7-mobiliario, dispuesto contra las paredes. Recuperado de Loude y Lièvre 1990.

nas. El porche del bashali está incluso menos estructurado, ya que no existe en él instalación alguna: ni hogares, ni elementos de asiento, ni estaciones de procesado... Si hubiésemos de encontrar restos de fuego de cocinado, éstos no estarían siempre en el mismo lugar.

Los interiores, por su parte, difieren enormemente. Frente a la rigidez del área de habitación, con su disposición pseudocircular, el bashali ofrece una habitación "vacía" que invita a la creatividad. En la casa menstrual, en vez de encontrar camas contra la pared, aparecen cuatro columnas en torno al fuego. No hay rastro de banquetas, ni altar central, ni onjesta-wao. Tampoco hay espacio dedicado al almacenaje ni contenedores. Aunque las que están en el bashali comen y duermen, la estancia principal de la casa menstrual funciona al margen de la vida diaria de la mujer kalasha.

La documentación etnográfica (Maggi 2001; Loude y Lièvre 1984; Lièvre y Loude 1990; Graziosi 1961) refuerza esta idea: las mujeres que residen en el bashali están libres de todas sus tareas: no van al campo, no cocinan ni cuidan de la casa o la familia, tampoco tejen o se encargan de los animales. En el bas- hali no se almacena, no se procesa ni se cocina. El alimento es traído por cada mujer cuando ingresa y/o es aportado por las visitas (que nunca entran). Todo aquello necesario para el aseo personal (peines, jabón etc.) se deja escondido entre las piedras del río o en los árboles, permanentemente dentro de las fronteras de la casa menstrual (Maggi 2001: 127). Los restos de comida y demás basura se queman en el hogar, pero no en el central, sino en otro situado en un rincón de la estancia (Graziosi 1961; Maggi 2001).

En dicho rincón radica la principal diferencia con el espacio habitacional: el carácter sacro del bashali. Esta zona es un espacio de culto dedicado a la diosa Dezalik. Según recogen Morgenstierne (1932), Graziosi (1961), Loude y Lièvre (1984) y Maggi (2001) Dezalik es considerada, junto con Dizau, una divinidad fundacional. Es también protectora de la fertilidad. Es a ella a la que se le hacen ofrendas especialmente cuando un parto se complica (ver Maggi 2001: 144-145). Es, por tanto, una divinidad de culto exclusivamente femenino. Las mujeres colocan ramas de roble, frutas, nueces y restos de comida sobre el altar de Dezalik o 


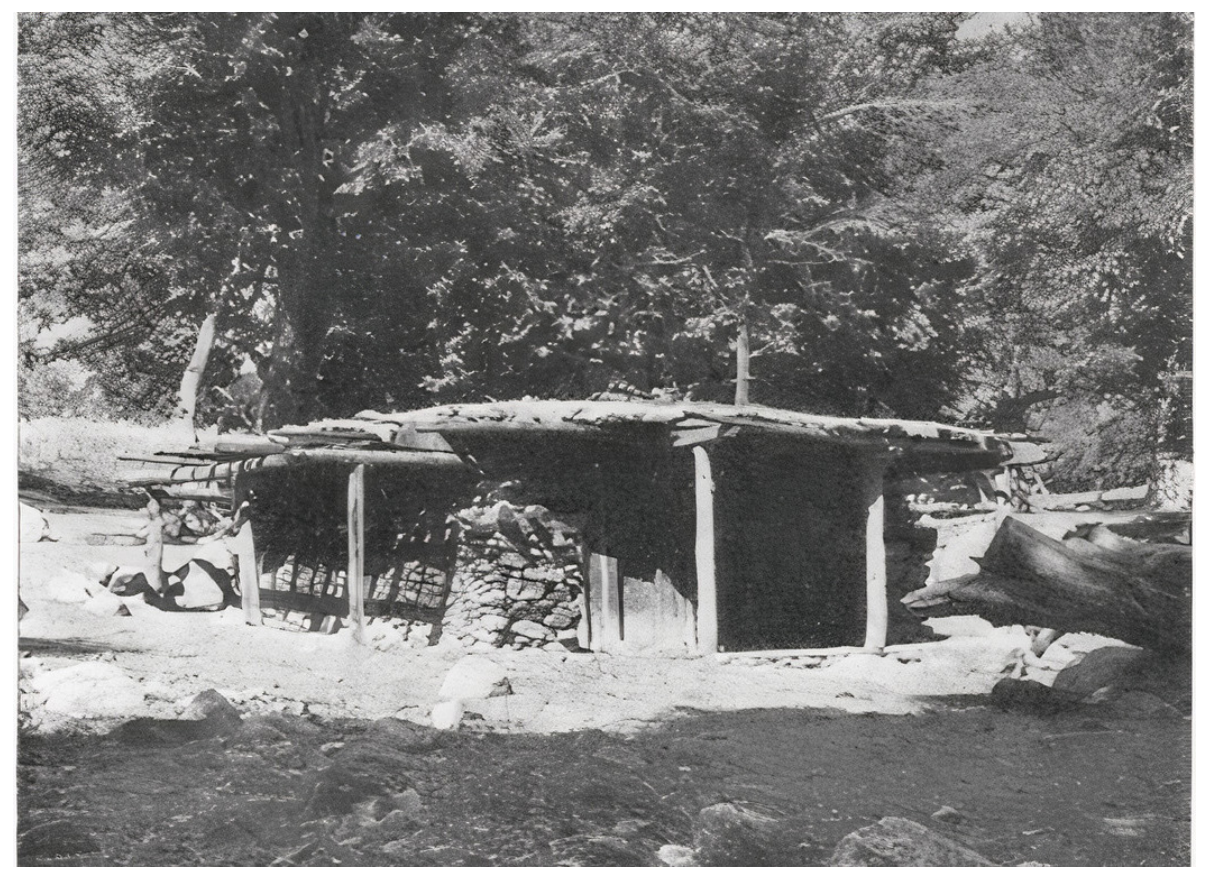

Figura 6. Fotografía del bashali del valle de Bumburet. Recuperado de Graziosi 1961.

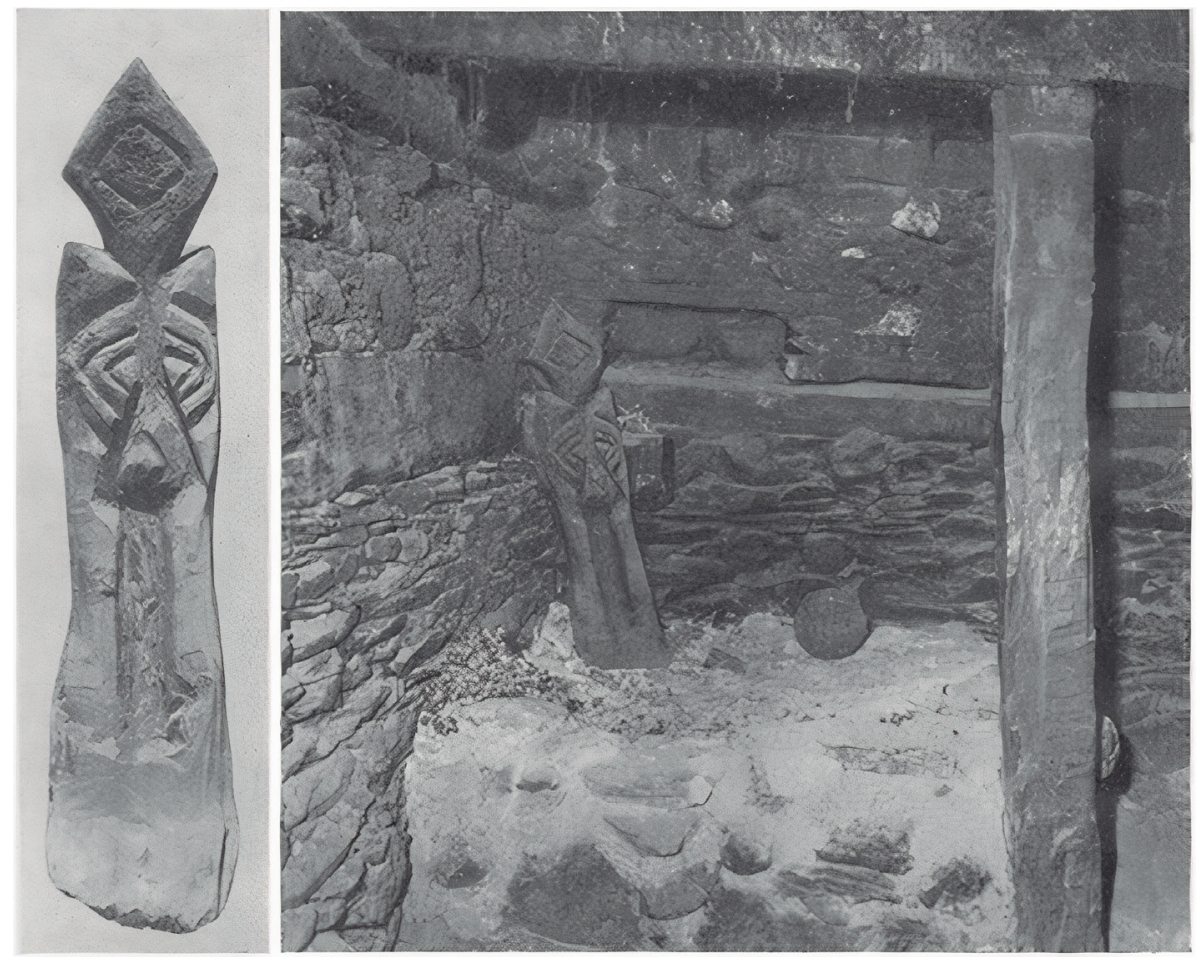

Figura 7. Fotografía del altar de Dezalik del bashali del valle de Bumburet y detalle de la talla de la diosa. Recuperado de Graziosi 1961.

los queman, como ya hemos mencionado, en el fuego que prenden en éste (Graziosi 1961; Maggi 2001). Sobre este mismo altar descansa también una imagen de madera: la talla de la diosa Dezalik (figura 7). El bashali, en definitiva, no solo contiene un espacio sacro, sino que es, en sí mismo, un santuario, hogar de una 
de las divinidades más importantes de la cultura kalasha.

\subsubsection{La casa menstrual y el espacio habitacional}

Como se puede desgajar de lo anterior, las diferentes materialidades de los espacios que nos ocupan (el doméstico y el menstrual) sirven para la creación de dos realidades bien diferentes. Por un lado, el espacio habitacional posibilita el desarrollo de cualquier actividad cotidiana y provee diferentes ambientes para el desarrollo de la vida diaria y las actividades de mantenimiento (Montón Subías 2011). Por otro, la desestructura de la casa menstrual posibilita un ambiente más distendido, pone la vida diaria en suspensión e introduce un uso para el culto.

Es especialmente significativa la desaparición del onjesta-wao, un elemento estructurante (Bafna 2003; Peponis y Wineman 2002) cuya existencia tiene un efecto normativo sobre una parte de los miembros del grupo: las mujeres. Este no sólo determina la forma en que una mujer se mueve por el espacio, sino que funciona como un dispositivo de dominación de género: en la interacción de ambos géneros existe un desequilibrio, creado a través de la materialidad espacial, que deja de ser necesario en aquellos espacios en que hombres y mujeres nunca conviven. Podemos, por tanto, entender el Onjesta-wao como un estructurante espacial normativo que existe específicamente para definir no la identidad femenina, sino la relación desigual entre los géneros.

Además de en la inexistencia del onjestawao, los espacios difieren en cuanto a la accesibilidad y la visibilidad. Mientras que por el emplazamiento (en forma aterrazada) y las condiciones arquitectónicas de la entrada (a través de una escalera), el espacio habitacional es enormemente privado, no existe barrera física alguna para entrar al bashali, ni siquiera está bien indicado dónde empieza el área pragata, haciendo la transición entre "fuera" y "dentro" enormemente orgánica y natural. Esta ausencia de barreras permite también una enorme visibilidad e intervisibilidad entre las mujeres que estén dentro y cualquiera que esté fuera de la casa menstrual, lo que no ocurre para el espacio doméstico que aparece como una zona más íntima.

En resumen, la casa menstrual de la tradición kalasha se presenta como inevitable por su posición junto al camino principal y perpetua por su accesibilidad durante todo el año. Además, es un elemento de cohesión comunal y aporta seguridad cuando se está "fuera de casa". Por añadidura, es un espacio de control sobre el camino, un lugar altamente accesible y una zona de gran visibilidad e intervisibilidad. Por otro lado, el bashali es la antesala de la sociedad kalasha tanto literal (porque es el lugar en que nacen los niños) como simbólicamente (pues recibe a todo el que quiere entrar en el valle).

Pero el bashali también es un lugar peligroso, sobre todo para las mujeres que se encuentran dentro. A pesar de pertenecer al mundo kalasha, en el bashali entran en juego otras reglas que implican que las personas fallecidas dentro no culminen satisfactoriamente el ciclo vital. Además de ambiguo y peligroso, el bashali ofrece una alternativa al orden social cotidiano posibilitando la creación de vínculos imposibles dentro del orden socio-familiar normal: cuando una mujer está de parto es atendida por las que se encuentran en el bashali. La que hace de partera, normalmente la de más edad, se convierte en la "madrina" de la criatura y le da al bebé unas cuentas que sustrae de su collar a través de las que se sella un vínculo familiar (Maggi 2001). Estos vínculos no se deshacen al abandonar el bashali, sino que se mantienen durante toda la vida y se pasan, junto con las cuentas de collar, de generación en generación. Por esto, podemos afirmar que, a pesar de que el bashali sea un espacio aparte, lo que ocurre impacta y modifica la realidad.

Por último, no nos podemos olvidar que, si bien en el bashali es relativamente fácil entrar, salir no lo es tanto. Abandonar el bashali sin el rito adecuado implica poner en peligro a toda la comunidad. No se puede dejar la casa menstrual sino mediante el agua, que permite la reintegración.

\section{La casa menstrual como espacio liminal}

Parece claro que el bashali dista bastante de la ponulu. Mientras que ésta última constituye un lugar de aislamiento, el bashali aúna todas y cada una de las características del espacio liminal (van Gennep 1960[1909]): un espacio que forma parte de la comunidad y, a la vez, queda fuera. A pesar de la apariencia lúdica y el ambiente distendido, el espacio liminal constituye un lugar peligroso, de cierta naturaleza sacra, cuyo abandono implica un rito de reintegración. 
Van Gennep (1960 [1909]), en su libro Les Rites de Passage, efectúa el primer análisis de los mecanismos que permiten a una persona avanzar en las diferentes fases de su existencia, de acuerdo a las categorías sociales dictadas por su cultura. A estos mecanismos, denominados ritos de paso, los reduce a una estructura tripartita: la separación, el margen o limen, y la agregación. En las sociedades orales, cada una de las partes del rito de paso se identifica con un lugar concreto, siendo la transición no sólo simbólica, sino también literal. Es entonces cuando el concepto de espacio liminal entra en juego: es una zona de ambigüedad que la persona en transición debe cruzar para abandonar su estado de partida y abrazar el de destino (ibid.: 15-25). La transición es, en palabras del propio van Gannep (ibid.: 22): "un rito espacial convertido en uno espiritual". Según esto, el espacio liminal no pertenece a la arena social, sino que es un lugar aparte, dominado por lo desconocido, lo sacro y lo ambiguo.

En el trabajo Edith y Victor Turner (1967; 1968; 1969; 1974; 1977a; 1977b; Turner y Turner 1978) la liminalidad se presenta como disparadora de la agencia individual y de la innovación social: "in liminality people "play" with the elements of the familiar (...) Novelty emerges from unprecedented combinations." (Turner 1974: 60). Para el matrimonio, lo liminal es un espacio de igualdad alejado de las instituciones mundanas donde existe un fuerte sentimiento de comunidad (Turner 1977a; Turner y Turner 1978). En él puede producirse un hermanamiento, una communitas que, dentro de la arena social, no habría sido posible.

Además del concepto de communitas, los Turner acuñaron muchos otros, fruto del estudio de la liminalidad en "sociedades modernas". Es precisamente este cambio de contexto y la colaboración con Richard Schechner la que sentará las bases para el desarrollo de los Performace Studies (Schechner 2013), que se centran en las sociedades "glocales", en las que la innovación social está en la liminalidad creada por la performance. A pesar de que lo liminal haya sido trabajado por diferentes autores y aplicado en diferentes contextos (ver Thomassen 2009), es la noción introducida por van Gennep, ligada a la estructura y el peligro, la que tiene mayor aplicación en sociedades orales (Thomassen 2012; Szakolczai y Thomassen 2019).

Así, si nos fijamos en el funcionamiento del bashali, podemos reconocer en él las caracte- rísticas del espacio liminal de van Gennep e identificarlo con el limen de un rito de paso que empieza con el ingreso de la mujer al bashali y acaba con su reintegración a través del agua. Este estado de liminalidad en que se encuentran los bebés de muchas culturas antes de su "bautizo" se extiende en el caso kalasha a las mujeres. Al igual que esos bebés, si fallecen, se entierran fuera de la necrópolis principal, las mujeres kalasha lo hacen en la necrópolis secundaria asociada al bashali.

La obra de van Gennep abre la puerta a entender el comportamiento ceremonial como clave de la lógica y la dinámica social (Huntington y Metcalf 1979: 11). El rito de paso (simbólico y material) funciona como un principio estructurante que, si bien pone las reglas en suspensión durante la transición, tiene un efecto normativo y ordenador. Así, el bashali, en tanto que espacio liminal, serviría para sustentar la lógica cultural y reforzar la desigualdad de género.

La imagen de las casas menstruales como espacios empoderantes ha sido, sin embargo, reivindicada por diferentes investigadoras cuyo trabajo se ha desarrollado en contextos de casas menstruales "abiertas" (aquellas percibidas por las mujeres como espacios de libertad (Maggi 2001: 240)). Este es el caso, por ejemplo, de Hitomi Tonomura y las ubuya, las casas de parto del japón premoderno, en cuyo análisis la autora encuentra "women's agency in giving meanings to their birthing process" (Tonomura 2007: 3). El argumento de la agencia femenina se ha estirado hasta sugerir que sería los hombres los que quedarían segregados del mundo de la mujer cuando esta integra la casa menstrual (Underhill 2015 [1936]). En este sentido va la diferenciación que hace Michelle Rosaldo (1974: 21) entre poder y autoridad culturalmente legitimada. Mientras que ésta última pertenece a los hombres, el poder puede ser ejercido por las mujeres gracias al peligro y al deseo que inspiran, desde lugares tradicionalmente considerados como desempoderantes: "the ideas of purity and pollution, so often used to circumscribe female activities, may also be used as a basis for assertions of female solidarity, power or value [...] Women may, for example, gather in menstrual huts, to relax or to gossip, creating a world free from control by men" (ibid.: 38).

Maggi (2001) también reconoce agencia femenina en el caso de la casa menstrual kalasha, de hecho, expone diversos casos de mu- 
jeres que deciden no ir al bashali en invierno o cuando están demasiado ocupadas. Además de esto, asocia a la casa menstrual toda una serie de experiencias positivas relacionadas con la formación de una comunidad femenina horizontal: las mujeres encuentran en el aislamiento menstrual una vía de escape a las estrictas normas de vestimenta femenina y las responsabilidades cotidianas y se dedican al aprendizaje e intercambio de información sobre salud, alimentación, belleza y cuidados, matrimonio y maternidad... Para Maggi, el bashali funciona como punto de encuentro entre mujeres de edades diferentes, de pueblos diferentes, de linajes y estatus diversos... sirviendo como pilar sobre el que construir la identidad femenina. Además de esto, desde el bashali se crean unos vínculos pseudo-familiares entre personas de diferente estatus y riqueza que refuerzan enormemente la cohesión social. El "espacio de libertad" (Maggi 2001: 9) que constituye el bashali se deriva, como hemos visto, de una configuración material que permite la suspensión de las reglas sociales cotidianas. Es, en definitiva, la (des)estructura la que propicia la creación de la communitas (Turner y Turner 1978) de la casa menstrual.

Sin embargo, no podemos dejar de ver en esta institución un elemento estructurante, no sólo de la vida de las mujeres, sino de la sociedad en general. En este sentido, el bashali se presenta como elemento regulador de las relaciones de género. Primero, ir al bashali es una parte importante del "ser mujer". Segundo, en tanto que ellas son garantes del equilibrio onjesta-pragata y sus movimientos se ven limitados, el bashali las inscribe en la relacionalidad (Hernando 2002). De hecho, la propia Maggi (2001: 166) transcribe las palabras de Saras Gula Aya, una mujer kalasha, amiga suya: "You know, young men are like that - they don't listen to just one woman. If you want them to do what you want, you have to join together". Para esta mujer la forma de ser escuchada era pertenecer a la comunidad de mujeres que se forma en el bashali. Tal y como apunta Michelle Rosaldo (1991: 75), "las mujeres obtienen poder y un sentido de importancia en cuanto son capaces de trascender los límites domésticos (...) creando una sociedad entre ellas".

Maggi (2001: 160-164) documenta como las mujeres kalasha, especialmente las jóvenes, usan el bashali como una vía de escape, a veces incluso ingresan en él sin tener el periodo, usando esta institución en su favor. Sin embar- go, cabe preguntarse, entonces, si realmente es posible ejercer el poder del que hablaba Michelle Rosaldo (1974) cuando se actúa en un "no espacio" o si deberíamos, más bien, clasificar los momentos de empoderamiento que ocurren en el bashali como una táctica (sen$s u$ de Certeau 1990), una argucia contra "una lógica simbólica regida por unas oposiciones binarias entro lo masculino y lo femenino que organizan ideal y materialmente (...) el mundo social” (Méndez 2016).

\section{Conclusión}

En este artículo hemos analizado el bashali, la casa menstrual de la cultura kalasha (Pakistán), a través de su materialidad en un intento de dar visibilidad a las mujeres y sus instituciones, que han sido tradicionalmente pasadas por alto en la literatura y siguen, a día de hoy, faltando en los discursos patrimoniales. En este sentido, reconocer la importancia de la casa menstrual kalasha y su contribución al mantenimiento cultural y social es crucial, especialmente ahora que los kalasha abandonan la oralidad y luchan por proteger y conservar su patrimonio. En efecto, esta cultura, como cualquier otra, no es estática sino cambiante $y$, en los últimos años, el cambio ha sido hacia el mundo de la tecnología, la escolarización, las redes sociales y el turismo. Ante esta situación, es imprescindible la preservación del patrimonio desde una perspectiva feminista.

En este sentido, se ha puesto de manifiesto que el bashali ha sido fundamental para la supervivencia de toda la comunidad ya que en tanto que espacio relacional, ha permitido salvaguardar las relaciones afectivas sin las que a supervivencia humana es imposible y, en tanto que espacio liminal ha sido motor de la dinámica social. El análisis de la materialidad ha sido especialmente útil a este respecto, ya que ha permitido desvelar significados que permanecen ocultos pero que conforman mecanismos muy potentes de cohesión grupal. A su vez, el estudio a través de la materialidad ha contribuido a superar la interpretación de la sangre menstrual como un fluido impuro y contaminante, rodeado de tabús y reintroducir su papel en la ordenación social y la inscripción de los individuos en el orden binario masculino-femenino.

Este trabajo ha permitido también "desuniversalizar" la imagen preconcebida de casa menstrual mediante el análisis de esta institución en un 
contexto concreto y su comparación con otros, de naturaleza bien diferente. Además, el texto permite poner sobre la mesa procesos biológicos como la menstruación, que están en la base de la esencialización y uniformización de las mujeres (Blázquez Rodríguez y Bolaños Gallardo 2017) y que en nuestra sociedad son un tabú.

Sin embargo, al poner el foco en la menstruación no debemos olvidar, tal y como escribe Guillo Arakistain (2013: 236), que "la regla está (...) en el centro del discurso biomédico hegemónico en torno al cuerpo femenino". En nuestras manos, el análisis de las casas menstruales debe ser menos una herramienta de esencialización (la menstruación como algo que hace mujeres) y más un útil que permita cuestionar dichos postulados. De esta forma, la reflexión desde la Academia, al igual que el activismo menstrual tiene el potencial de "undermine gender as a stable category" (Bobel 2010: 12). En definitiva, entender la reflexión histórico-arqueológica en clave feminista implica la preservación de un patrimonio que, tradicionalmente, no ha sido valorado ni visibilizado y, desde ahí, el emprendimiento de una reflexión con vistas a conseguir un futuro más justo.

\section{Bibliografía}

Ahlrichs, J. J.; Riehle, K.; Sultanalieva, N. (2018): The Production of Liminal Places: An Interdisciplinary Account. Ethnographisch-Archäologische Zeitschrift 56(1,2): 205-242.

Ali, M. K.; Chawla, M. (2019): Socio-Cultural Life of Kalasha People of Chitral: A Study of their Festivals. Pakistan Vision, 20(2): 42-57.

Ali, S. S.; Rehman, J. (2001): Indigenous Peoples and Etnic Minorities of Pakistan. Institutional and Legal Perspectives. Curzon Press, Londres.

Arrieta Urtizberea, I. (2017): El sesgo androcéntrico en el patrimonio cultural. El género en el patrimonio cultural (I. Arrieta Urtizberea, ed.), Universidad del País Vasco, Bilbao: 11-18.

Babel, C. (2010): New Blood: third-wave feminism and the politics of menstruation. Rutgers University Press, Nueva Brunswick, Nueva Jersey y Londres.

Bafna, S. (2003): Space syntax: a brief introduction to its logic and analytical techniques. Environment and Behavior, 35(1): 17-29. https://doi.org/10.1177/0013916502238863.

Birriel Salcedo, M. M.; Rísquez Cuenca, C. (2016): Patrimonio, turismo y género. Estrategias para integrar la perspectiva de género en el patrimonio histórico. Revista $\mathrm{PH}$ : Boletín del Instituto Andaluz del Patrimonio Histórico 89: 128-133. https://doi.org/10.33349/2016.0.3753.

Blake, J. (2015): Género y patrimonio cultural inmaterial. Igualdad de género: patrimonio y creatividad (UNESCO, ed.), UNESCO, París: 48-59.

Blázquez Rodríguez, M. y Bolaños Gallardo, E. (2017): Aportes a una antropología feminista de la salud: el estudio del ciclo menstrual. Salud Coelctiva 13(2): 253-265. https://doi.org/10.18294/sc.2017.1204

Buckley, T.: Gottlieb, A. (1988): A Critical Appraisal of Theories of Menstrual Symbolism. Blood Magic: The Anthropology of Menstruation (T: Buckley; A. Gottlieb, ed.). University of California Press, Berkeley: 3-53.

Cacopardo, A. (2016a): Fence of Peristan. The Islamization of the "Kafirs" and Their Domestication. Archivio per l'Antropologia e La Etnologia 145: 69-101.

Cacopardo, A. (2016b): Pagan Christmas. Winter Feasts of the Kalasha of the Hindu Kush. Gingko Library, Londres.

Cacopardo, A.; Cacopardo, A. (2001): Gates of Peristan: History, Religion and Society in the Hindu Kush. Istituto Italiano per I'Africa e I'Oriente, Roma.

Claassen, C., (2011): Rock shelters as women's retreats: understanding Newt Kash. American Antiquity, 76(4): 628-641. https://doi.org/10.2307/41331915.

Darling, E. G. (1979): Merit Feasting among the Kalash Kafirs of Northwestern Pakistan (Tesis de Máster). University of British Columbia, Vancouver.

De Certeau, M. (1990): L'invention du quotidien 1. Arts de faire. Gallimard, Paris

di Carlo, P. (2007): The Prun Festival of Birir Valley, Pakistan, in 2006. East and West, 57(1-4): 45-100. https://doi.org/10.2307/29757723.

Douglas, M. (1973): Pureza y Peligro. Siglo Veintiuno Ediciones, Madrid.

Engelstad, E. (1991): Images of power and contradiction: feminist theory and post-processual archaeology. Antiquity 65(248): 502-514. https://doi.org/1017/S0003598X00080108 
Faust, A.; Katz, H. (2017): The Archaeology of Purity and Impurity: A Case-Study from Tel 'Eton, Israel. Cambridge Archaeological Journal, 27(1): 1-27. https://doi.org/10.1017/S0959774316000494.

Fernández Valencia, A. (2005): Las mujeres como sujeto histórico: género y enseñanza de la historia. Didáctica de las Ciencias Experimentales y Sociales 18: 5-24.

Frandsen, P.J. (2007): The menstrual 'taboo' in Ancient Egypt. Journal of Near Eastern Studies, 66(2): 81-106. https://doi.org/10.1086/519030.

Fries, J.; Mattias, J.; Rambuscheck, U.; Gutsmiedl-Schümann, D. (2017): Images of the Past. Gender and its Representations. Waxmann, Münster, Nueva York.

Galloway, P. (1998): Where have all the menstrual huts gone? The invisibility of menstrual seclusion in the late prehistoric southeast. Reader in Gender Archaeology (K. Hays-Gilpin y D. Whitley, eds.) Routledge, London/New York: 197-211.

García Luque, A. (2015): Mujeres visibles e invisibles en la enseñanza de la Historia de la Educación Primaria: cambios y pervivencias al amparo de la LOMCE. Una enseñanza de las Ciencias Sociales para el futuro: recursos para trabajar la invisibilidad de personas, lugares y temáticas (A. M. Hernández, C. R. García Ruíz y J. L. de la Montaña, eds.), Universidad de Extremadura, Cáceres: 165-173.

García Luque, A. (2016): Incorporar la perspectiva de género en la enseñanza-aprendizaje de la historia: un desafío didáctico y formativo. Revista PH: Boletín del Instituto Andaluz del Patrimonio Histórico 24(89): 147-149. https://doi.org/10.33349/2016.0.3739.

Geertz, C. (1973): The Interpretation of Cultures. Basic Books, New York.

GHRD (2020): Tribe of Kalash: The Last Kafir. Global Human Rights Defense (ECOSOC-Naciones Unidas) Sección Noticias. Recuperado de: https://www.ghrd.org/2021/03/01/tribe-of-kalash-the-last-kafir/

Gifford-Gonzalez, D. (1993): You Can Hide, But You Can't Run: Representations of Women's Work in Illustrations of Palaeolithic Life. Society for Visual Anthropology Newsletter 9(1): 22 - 4. https://doi. org/10.1525/var.1993.9.1.22.

Gonzalez Marcén, P. (Coord.) (2012): Los trabajos de las mujeres y el lenguaje de los objetos. Recuperación de la cultura material femenina como herramientas de transmisión de valores. Memoria del proyecto $i+d+i 2007$ exp.: 002/07 (2007-2010) financiado por el Instituto de La Mujer. Universidad Autónoma de Barcelona, Barceclona.

Graziosi, P. (1961): The Wooden Statue of Dezalik, a Kalash Divinity, Chitral, Pakistan. Man, 61: 148-151. https://doi.org/10.2307/2796942.

Guillo Arakistain, M. (2013): La in-corporación de la investigación. Políticas de la menstruación y cuerpos (re)productivos. Nómadas 39: 233-245.

Hernando A. (2002): Arqueología de la Identidad. Akal, Madrid.

Huntington, R.; Peter Metcalf, P. (1979): Celebrations of Death: The Anthropology of Mortuary Ritual. Cambridge University Press, Cambridge.

Jettmar, K. (1961): Ethnological research in Dardaristan 1958. Preliminary report. Proceedings of the American Philosophical Society 105(1): 79-97. https://doi.org/10.2307/985355.

Jiménez-Esquinas, Guadalupe. (2016): De “añadir mujeres y agitar" a la despatriarcalización del patrimonio: la crítica patrimonial feminista. Revista PH, 89: 137-140. https://doi.org/10.33349/2016.0.3708.

Jiménez-Esquinas, G. (2017): El patrimonio (también) es nuestro. Hacia una crítica patrimonial feminista. El género en el patrimonio cultural (I. Arrieta Urtizberea, ed.). Universidad del País Vasco, Bilbao: $19-48$.

Jiménez-Esquinas, G. (2021): Género: cómo revertir las desigualdades desde los museos. Patrimonio y museos locales: temas clave para su gestión (I. Arrieta Urtizberea e I. Díaz Balerdi, eds.) PASOS, RTPC, Tenerife: 295-308.

Junaidi, I. (2017): Rights activists highlight threats to existence of Kalash tribe. Dawn. Recuperado de https://www.dawn.com/news/1335490

Lièvre, V. ; Loude, J. Y. (1990): Le Chamanisme Des Kalash Du Pakistan : Des Montagnards Polythéistes Face À L'islam. Editions Recherche sur les Civilisations, Paris.

Lièvre, V.; Loude, J. Y. (1980): Kalash: Les derniers infidèles de l'Hindu-Kush. Berger-Levrault, Paris.

Loude, J. Y. ; Lièvre, V. ; Nègre, H. ; Maury, H. (2018): Fêtes Himalayennes. Les derniers kalash. La boîte à bulles y Musée des confluences, Saint-Avertin y Lyon.

Loude, J. Y.; Lièvre, V. (1984): Solstice Païen. Presses de la Renaissance, Paris.

Maggi, W. (2001): Our Women are Free. Gender and Ethnicity in the Hindu Kush. The University of Michigan Press, Ann Arbor. 
Malik, J. A.; Waheed, A. (2005): KALASH: The challenge of development with identity. Meanings and Issues. Institute of Social Policy, Islamabad.

Méndez, L. (2007): Antropología feminista. Síntesis, Madrid.

Méndez, L. (2016): Mucho más que un signo de impureza: el sexo que sangra en clave antropológica. Letras escarlata. Estudios sobre a representación da menstruación (T. Bermudez; M. H. Carvalho de Sant'Anna, eds.) Frank \& Timme, Madrid: 43-60.

Montón-Subías, S. (2011): Las Actividades de Mantenimiento en la Arqueología del Género en España. Mujeres: Miradas Interdisciplinarias (M. J. Rodríguez-Shadow; L. Campos Rodríguez, eds.) Centro de Estudios de Antropología de la Mujer, Mexico DF: 268-286.

Morgenstierne, G. (1932): Report On A Linguistic Mission To North-Western India. Instituttet For Sammenlignende Kulturforskning, Oslo.

Naqvi, F. H. (1996): People's Rights or Victim's Rights: Reexamining the Conceptualization of Indigenous Rights in International Law. Indiana Law Journal 71(3): 674-728.

Navarro Rodríguez, E. (2018): Análisis de los discursos y las imágenes en los museos arqueológicos desde una perspectiva feminista: estudio de tres casos. Arqueología y Territorio 15: 139-151.

Oestigaard, T. (2005): Water and World Religions: An introduction. SFU \& SMR, Bergen.

Oestigaard, T. (2011): Water. The Oxford Handbook of the Archeology of Ritual and Religion (T. Insoll, ed.). Oxford University Press, Oxford: 76-88.

Ortner, S. B. (1974): Is female to male as nature is to culture?. Woman, culture, and society (M. Z. Rosaldo; L. Lamphere, eds.), Stanford University Press, Stanford: 68-87.

Parkes, P. (1987): Livestock Symbolism and Pastoral Ideology among the Kafirs of the Hindu Kush. Man, 22(4): 637-660. https://doi.org/10.2307/2803356.

Parkes, P. (2000): Enclaved knowledge: Indigent and indignant representations of envirinmental management and development among the Kalasha of Pakistan. Indigenous Enviromental Knowledge and its Transformations: Critical Anthropological Perspectives (A. Bicker; R. Ellen; P. Parkes, eds.) Routledge, Londres: 253-292

Peponis, J.; Wineman, J. (2002): Spatial structure of environment and behavior. Handbook of environmental psychology (R. Bechtel y A. Churchman eds), John Wiley and sons Inc., New York: 271-291.

Querol, M. A. (2007): El tratamiento de las mujeres en las reconstrucciones prehistóricas: nuevos relatos para el siglo XXI. Boletín de la Real Academia de Extremadura de las Letras y las Artes 15: 201-212.

Querol, M. A. (2014): Museos y Mujeres: la desigualdad en Arqueología. Arqueoweb: Revista sobre Arqueología en Internet 15(1): 270-280.

Robertson, G. (1896): The Kafirst of the Hindu Kush. Lawrence and Bullen, Londres.

Rosaldo, M. Z. (1974): A Theoretical Overview. Woman, Culture, and Society (M. Z. Rosaldo; L. Lamphere, eds.) Stanford University Press, Stanford: 17-42.

Rosaldo, M. Z. (1991): Mujer, cultura y Sociedad. Una visión teórica. Antropología y Feminismo (O. Harris; K. Young, comp.) Cátedra, Madrid: 153-180.

Rose, C. (1992): Progress and Culture. The kalasha struggle to survive. Institute of Current World Affairs. Recuperado de https://www.icwa.org/carol-v-rose-newsletters/

Sánchez Romero, M. (2019): La construcción de los discursos sobre las mujeres en el pasado: las aportaciones de la arqueología feminista. Paradigma 22: 92-95.

Schomberg, R. C. F. (1938): Kafirs and Glaciers. Travels in Chitral. Hopkinson, Londres.

Sherfield, A. (2019): Archaeology of Menstruation. Anthropology News website, 17 de julio. https://doi. org/10.1111/AN.1224.

Siiger, H. (1956): Ethnological Field Research in Chitral, Sikkim, and Assam: A Preliminary Report. Historiskfilologiske Meddelelser udgivet af Det Kongelige Danske Videnskabernes Selskab, 36(2): 12-34.

Smith, L. (2008): Heritage, Gender and Identity. The Ashgate Research Companion to Heritage and Identity (B. Graham y P. Howard, eds.), Ashgate Publishing, Farnham: 159- 178.

Strassmann, B. (1992). The function of menstrual taboos among the dogon: Defense against Cuckoldry?. Human Nature, 3(2): 89-131. https://doi.org/10.1007/BF02692249.

Strassmann, B. (1996): Menstrual hut visits by Dogon women: a hormonal test distinguishes deceit from honest signaling, Behavioral Ecology, 7(3): 304-315. https://doi.org/10.1093/beheco/7.3.304.

Strassmann, B.; Kurapati, N.; Hug, B.; Burke, E.; Gillespie, B.; Karafet, T.; Hammer, M. (2012): Religion as a means to assure paternity. Proceedings of the National Academy of Sciences of the United States of America, 109(25): 9781-9785. https://doi.org/10.2307/41602752. 
Szakolczai, A.; Thomassen, B. (2019): Victor Turner: Liminal Experiences as the Grounding of Social Theory. From Anthropology to Social Theory: Rethinking the Social Sciences (B. Thomassen y A Szakolczai, eds.), Cambridge University Press, Cambridge: 176-196. doi:10.1017/9781108529426.008. Thomassen, B. (2009): The Uses and Meanings of Liminality. International Political Anthropology, 2.1: 5-27. http://www.politicalanthropology.org/.

Thomassen B (2012): Revisiting liminality: the danger of empty spaces. Liminal Landscapes. Remapping the Field (H. Andrews y L. Roberts L, eds.). Routledge, Londres: 21-35. https://doi. org/10.4324/9780203123164.

Tonomura, H. (2007): Birth-giving and Avoidance Taboo: Women's Body versus the Historiography of Ubuya. Nichibunken Japan review : journal of the International Research Center for Japanese Studies 19: 3-45. https://doi.org/10.15055/00000227

Turner, V. (1967): Betwixt and Between: The Liminal Period in Rites de Passage. The Forest of Symbols: aspects of Ndembu ritual. Cornell University Press. Londres e Ithaca: 93-11.

Turner, V. (1968): The drums of affliction: A study of relgious processes among the Ndembu of Zambia. Cornell University Press, Ithaca.

Turner, V. (1969): The Ritual Process: Structure and Anti-structure Aldine, Chicago.

Turner, V. (1974): Liminal to Liminoid, in Play, Flow, and Ritual: An Essay in Comparative Symbology. Rice University Studies 60(3): 53-92.

Turner, V. (1977a): The ritual process: structure and anti-structure. Cornell University Press, Ithaca.

Turner, V. (1977b): Variations on a Theme of Liminality. Secular Ritual (S. Moore y B. Myerhoff, eds.). Van Gorcum, Amsterdam: 36-52.

Turner, V.; Turner, E. (1978): Image and pilgrimage in Christian culture. Columbia University Press, New York.

Underhill, R. 2015 [1936]: The Autobiography of a Papago Woman. Martino Publishing Centre, Mansfield. Van Gennep, A. (1960) [1909]: The rites of Passage. University of Chicago Press, Chicago. 